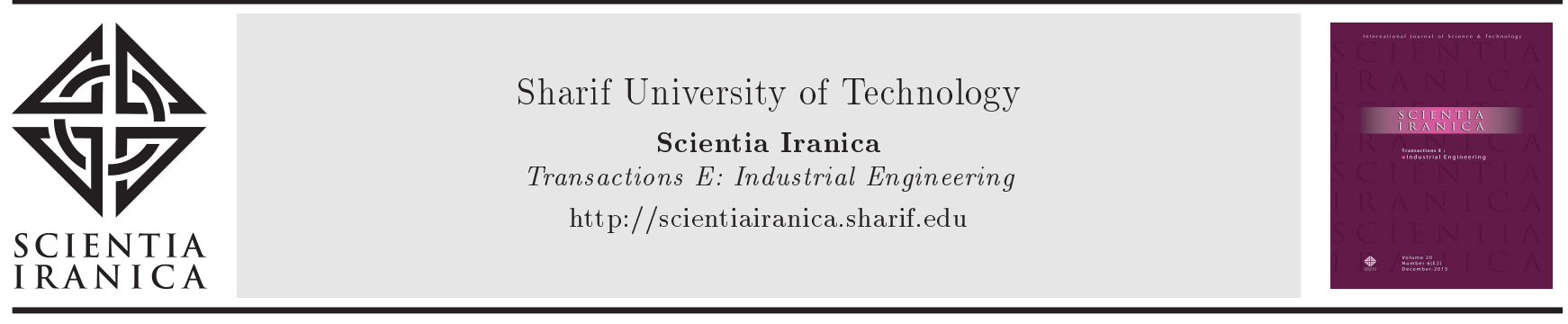

\title{
Demand-driven condition-based maintenance planning under Markovian deterioration of machine condition
}

\author{
R. Noori, A. Sadegheih*, and M.M. Lotfi \\ Department of Industrial Engineering, Yazd University, Yazd, P.O. Box 98195-741, Iran. \\ Received 26 July 2019; received in revised form 7 December 2020; accepted 1 February 2021
}

\author{
KEYWORDS \\ Inspection planning; \\ Demand-driven; \\ Condition-based \\ maintenance; \\ Stochastic-dynamic \\ process; \\ Minimal repair.
}

\begin{abstract}
In this paper, single-product single-machine systems under Markovian deterioration of machine condition throughout a specified finite planning horizon are studied. It is assumed that a machine is subject to random failures and that any maintenance activities applied to it in a period reduce the system's potential production capacity during that period. Furthermore, it is assumed that the machine is minimally repaired upon failure, and PM is carried out, after inspection, to restore the machine to the 'as-good-as-new' status. The objective of the study is to find the optimal intervals for inspection and Preventive Maintenance (PM) activities in Condition-Based Maintenance $(\mathrm{CBM})$ planning with a discrete monitoring framework subject to minimize the sum of inspection, PM, minimal repair, and backlog costs. To this end, a stochastic dynamic programming model that enumerates demand is presented, called the demand-driven CBM model. The numerical results show that this model significantly decreases the total cost which depends on the demand and the unit backlog cost, which is an increasing and concave function in the unit backlog cost regardless of the initial machine state.
\end{abstract}

(C) 2023 Sharif University of Technology. All rights reserved.

\section{Introduction}

In recent decades, the approach to Just In Time (JIT) in production and operation management as well as the production procedures has transformed and JIT roles have become more prominent. Machine deterioration is one of the main reasons for production capacity loss and consequences of delay in customer requests in many manufacturing industries. Promotion of machine health through preventive maintenance is a policy that aims to restore production capacity which, in turn, enhances the timely delivery of customer

\footnotetext{
*. Corresponding author. Tel.: +9835 31232402;

Fax: +983531232403

E-mail addresses: rezanoori84@gmail.com (R. Noori); sadegheih@yazd.ac.ir (A. Sadegheih); lotfi@yazd.ac.ir (M.M. Lotfi).
}

doi: $10.24200 /$ sci.2021.54111.3597 demands [1]. However, preventive maintenance reduces machine unavailability and, thus, increases the potential production capacity of the machine for processing customer demands. Therefore, adopting a preventive maintenance strategy that can keep the machine well enough to meet customer demands is necessary [2].

Generally, in literature, there are two strategies for Preventive Maintenance (PM), namely Time-Based Maintenance (TBM) and Condition-Based Maintenance (CBM). Traditionally, PM is carried out in the form of system overhaul or unit replacement based on the elapsed time, which is often mentioned as timebased maintenance. TBM policies are usually approved based on a probabilistic model of system failure. In TBM, the machine age is the basis of the planning and the maintenance is carried out after a specific period of time regardless of the health status of a physical asset and customer demand. In this approach, employing a suitable policy to determine PM durations and the 
frequencies is very important because an over frequent policy leads to additional cost and an over duration policy leads to unexpected failures $[3,4]$.

In recent years, to reduce the number of unnecessary scheduled preventive maintenance operations and eliminate the risks associated with them, more efficient maintenance approaches such as conditionbased maintenance have emerged [5]. Unlike TBM policies built on historical failure data, CBM is a maintenance approach that emphasizes the information collected through condition monitoring.

This policy consists of two stages. In the first stage, the system status is evaluated and the machine state is identified. This stage can be either a continuous monitoring (inspection) or a discrete one. In continuous monitoring, which may be costly in some industries, some sensors continuously control the system status. Through continuous monitoring, one continuously monitors (usually by mounted sensors) a machine and triggers a warning alarm whenever something goes wrong. Continuous monitoring is subject to two limitations. Firstly, it is often expensive and secondly, it produces inaccurate diagnostic information due to the monitoring of raw signals with noise, continuously [6]. In discrete monitoring, some machine's significant covariates will be measured within a specific period of time. Although this approach is more economical than the continuous one, there is always the risk of missing some alert between two inspections. In this type of monitoring, if the inspection is over frequent, the inspection cost will increase; in contrast, the cost of unnecessary PM operation is reduced. In contrast, if the inspection frequency decreases, the total cost of PM operation and unexpected failure will increase despite the decrease in inspection cost. Accordingly, there are different approaches to determining the duration and frequency of inspections in the discrete monitoring approach. The first one is to do it at a fixed interval. However, in the second approach, the duration is constant, but frequency is achieved by running a model considering different economic measures. In the third approach, the duration is not constant and the frequency is not predefined; in fact, it extracts an optimal plan using a suitable model [7-10]. Furthermore, in [11], all possible inspection schemes were incorporated to prevent local optimum solutions and avoid unnecessary combinations. In [12], the possibility to moderate or reduce the number of inspections undertaken was considered. As such, a proportional hazard model was used for the risk of failure and a Markovian process was employed to model the system covariates. In the second stage, based on the collected information from the first stage, the PM execution interval is determined. In CBM, the PM operations are executed if the machine's significant covariates are more than predefined values called threshold. The main challenge in CBM is to identify the optimum threshold policy for PM execution. In [13,14], a maintenance policy for a degrading system with ageand state-dependent operating costs was proposed in which cost increased with the aging of the system and degradation levels. Authors in [15] proposed a partially observable Markov decision process structure in which optimal inspection and maintenance strategies must be adopted to maximize both the system availability and the expected value of profit.

In the optimization procedure of the previous models, customer requirements is neglected and the modeling is implemented based on the hazard rate function of the machine. In the following, some models are investigated in which production and PM planning are integrated. In [16,17], simultaneity production and maintenance planning for a multi-product single-machine construction system were modeled as a Markov decision process in which the machine deteriorated. The objective was to determine the machine maintenance plan and the production amount simultaneously so that the sum of expected production, backorder, and holding costs could be minimized. In $[18,19]$, a semi-Markov decision process model for a single-stage production system with multiple products and multiple maintenance actions was presented. In [2,20,21], other factors such as the backlog cost were added to previous factors to run the optimization model for threshold. None of these models have considered inspection as a decision variable and the cost of inspection, although they assumed other decisions involved in production planning such as production, inventory, and backorder quantity.

To the best of our knowledge, there has been no model that has determined inspection and preventive maintenance planning simultaneously in the presence of the Markovian deterioration of machine condition and customer's demand. However, in practice, demand motivates running the machine. For example, consider a situation in which the optimum time for PM execution coincides with the customer order reparation and delivery. This coincidence leads to a delay in order delivery and, accordingly, customer dissatisfaction. Therefore, delay in PM operations results in unexpected failure which translates into undesired effect on the backlog cost and customer's satisfaction.

The key contributions of this paper are as follows:

1. Presenting a new model for condition-based maintenance with discrete monitoring whose objective is to find inspection and PM interval in the presence of the Markovian deterioration of machine condition and customer's demand;

2. The ability of the presented model to consider unexpected failures;

3. Demonstrating that the total cost would be reduced 
significantly by enumerating customer demand in the CBM model. Although the direct CBM costs counting inspection, preventive maintenance and, minimal repair might increase by using this model, the total cost would reduce.

To this end, by employing stochastic dynamic programming, a model for condition-based maintenance planning at a tactical level in a finite time horizon is presented. The model consider discrete inspections whose frequencies are neither predefined nor fixed, but they are decision variables. Moreover, based on the result of the inspection, the optimum interval for PM, which depends on the state variable whose value emerges during the inspection stage, will be determined. In this model, demand is certain, while the machine status is not certain. This uncertainty is defined by considering the machine status as a state variable, and the related decision variables are calculated such that the sum of inspection, PM, minimal repair, and backlog costs is minimized. This model is run for all possible inspection plans $\left(2^{K}\right.$ possibilities, where $k$ equals the number of periods). The results are compared and the optimal combination of inspection and PM time is found. Furthermore, to analyze the effect of enumerating customer demand on total cost, an illustrative example is designed and investigated.

This paper is structured as follows. Section 2 explains the problem and its corresponding assumptions in general. Section 3 describes our modeling framework including appropriate mathematical representation. Section 4 gives a solution algorithm. Section 5 describes numerical study and its computational sequences. Finally, Section 6 concludes this paper.

\section{Problem statement}

In this research, a single-product system is considered and condition-based maintenance planning is achieved in a finite-time horizon with $K$ equal intervals with a length of $T$. It is assumed that $\Delta$ consisting of $K$ elements is the demand in the planning horizon. Its elements show the demand in different periods at the beginning of the planning horizon. The demand for every period must be satisfied within the same period; otherwise, for every unit of the unsatisfied demand, a value represented by $h$ will be lost. For simplicity, the whole production system is considered as a single machine that deteriorates during its operation time because of production. A variety of levels are defined for the machine deterioration, called state, and the set of machine states is shown by $\mathbb{S}=\{0,1,2, \cdots, N\}$. The deterioration is assumed to be a Markov stochastic process, i.e., if $\mathcal{X}=\left(X_{t}: t \in[0, \infty)\right)$ is the machine deterioration process and assuming that $\mathcal{X}$ is a homogeneous continuous-time Markov process with discrete state space $\mathbb{S}$, then the deteriorating machine can be in one of $N$ operational states $0,1,2, \cdots, N-1$ or a failure state $N$. The machine has the best performance in the state 0 and is out of service in the state $N$. In other states, a larger number shows a lower performance; hence, $r(s)$ is an absolutely decreasing function while $r(s)$ is the machine performance in the state $s$.

As mentioned before, a model is proposed in the stochastic dynamic programming form for overcoming this problem. This model consists of two stages: The first stage is related to inspections, while the second one includes decision-making about appropriate intervals for PM activities.

In the first stage, it is assumed that the machine state is observable in the inspections, i.e., its specification is error-free (in contrast with the hidden state). Moreover, it is assumed that at the beginning of the planning horizon, the inspection must be executed. The successive inspection is carried out only at the beginning of each period while conducting the inspections depends on our decision. The inspection processing time and cost are constant parameters and are denoted by $c_{i n s}$ and $t_{i n s}$, respectively.

In the second stage, it is assumed that the machine state becomes zero, i.e., 'as-good-as-new' status, merely following the execution of PM operation. PM cost and processing time, which depend on machine state $(s)$, are absolutely increasing functions in $s$. These parameters are denoted by $c_{p m}(s)$ and $t_{p m}(s)$, respectively. Moreover, we assume that $c_{p m}(0)$ and $t_{p m}(0)$ are equal to zero. Furthermore, within a period, if the machine reaches the failure state, then it is assumed to be minimally repaired and restored to $N-1$ state, i.e., 'as-bad-as-old' status, immediately. Minimal repair cost and its processing time are constant parameters and are denoted by $c_{m r}$ and $t_{m r}$, respectively.

The inspection plan identifies the inspection frequency and, therefore, has a direct impact on inspection cost. However, it has an indirect impact on other costs because other procedures will be carried out after inspection execution and they depend on the inspection plan. In addition to the inspection cost, there are three types of other costs. The first type is the PM cost; the second type is the minimal repair cost; and the third type is the backlog cost due to machine unavailability during the inspection, PM, or minimal repair operations. To consider this cost, besides creating a dependency between production rate and machine state, the time elapsed for inspection, $\mathrm{PM}$, and minimal repair operations is also taken into account. This cost is proportional to the difference between actual production capacity and the demand in each period. The objective is to find the optimal intervals for inspection and $\mathrm{PM}$ activities in $\mathrm{CBM}$ planning with a discrete monitoring framework over a finite time horizon to satisfy the demand. Here, 
the appropriate PM interval must be based on the inspection results so that the total cost in the planning horizon, including inspection, PM, minimal repair, and backlog cost, is minimized.

\section{The mathematical model}

This section describes a basic model of stochastic dynamic programming in the finite time horizon to develop our model and, then, presents the proposed model along with a detailed modeling framework.

\subsection{Basic model of stochastic dynamic programming with finite time horizon}

Here, stochastic dynamic programming with finite-time horizon method is used for modeling the problem. In this method, deriving the optimality equation as a backward recursive equation is the most important part of the modeling. The components of this equation include stage $(n)$, state variable $(s)$, action $(a)$, transition probability matrix $(P(a))$, and current stage cost $(C(s, a))$ when the system state is $s$ and action $a$ is decided. Eq. (1) shows the optimality equation in the general form:

$$
V_{n}(s)=\min _{a \in \mathbb{A}}\left\{C(s, a)+\sum_{s^{\prime} \in \mathbb{S}} P_{s s^{\prime}}(a) V_{n-1}\left(s^{\prime}\right)\right\}
$$

$(\forall n \geq 1)$,

where $\mathbb{S}$ and $\mathbb{A}$ are state and action spaces, respectively, and $V_{n}(s)$ shows the expected total cost while the system state is $s$ and there are $n$ stages to the end of the planning horizon. Moreover, it is assumed that $V_{0}(s)=0(\forall s \in \mathbb{S})[22]$.

\subsection{Proposed model with customer demand}

In this section, the problem is modeled under a stochastic dynamic programming framework with emphasis on the customer demand. For this purpose, first, the optimality equation components are defined and, then, the optimality equation is established.

\subsubsection{Components of the proposed model}

\section{The first component (stage)}

Each point of decision about the inspection is defined as a stage. The model structure needs to be given number of the remaining periods to the end of the planning horizon in each stage. The beginning of each period is an option for the inspection; therefore, the inspection in each period is considered as a binary decision variable. Each feasible solution is an array that consists of 0 and $1 s$ which is shown by $I=\left(i_{k}\right)_{k=0}^{K}$ and referred to as inspection plan. It is assumed that an inspection is performed at the beginning of the first period. Moreover, for simplicity, we assume that the last element of each sequence is 1 . That is, each sequence starts and ends with 1 , where 1 at the end of the sequence is not a real inspection and is only for the simplicity of modeling, i.e.:

$$
I=\left(i_{0}, i_{1}, \cdots, i_{K}\right)=\left(i_{k}\right)_{k=0}^{K}, \quad i_{0}=i_{K}=1,
$$

where $K$ is the number of periods included in the planning horizon and the set of feasible solutions is shown by $\mathbb{I}$. Therefore, the number of $\mathbb{I}$ elements is equal to $2^{K-1}$.

Assume that sequence $I=\left(i_{k}\right)_{k=0}^{K}$ is an arbitrary inspection plan and henceforth constant, and assume that $\bar{I}$ is a subsequence of $I$ that consists of its 1 s, i.e.:

$$
\bar{I}=\left(\bar{i}_{k_{0}}, \bar{i}_{k_{1}}, \cdots, \bar{i}_{k_{m(I)}}\right)=\left(\bar{i}_{k_{i}}\right)_{i=0}^{m(I)}
$$

such that:

$$
\bar{i}_{k_{i}}=1 \quad \forall i \in\{0,1, \cdots, m(I)\},
$$

and:

$$
i_{k}=0 \quad \forall k \notin\left\{k_{0}, k_{1}, \cdots, k_{m(I)}\right\} .
$$

Now, the sequence $J(I)=\left(j(I)_{n}\right)_{n=0}^{m(I)}$ is defined as follows:

$$
\begin{aligned}
J(I) & =\left(j(I)_{0}, j(I)_{1}, \cdots, j(I)_{m(I)}\right) \\
& =\left(K-k_{m(I)}, K-k_{m(I)-1}, \cdots, K-k_{1}, K-k_{0}\right) .
\end{aligned}
$$

The sequence $J(I)$ indirectly identifies the stages called stages sequence. The number of sentences in this sequence determines the number of stages.

Example 1. Assume that $I=(1,0,0,1,0,1,1)$ is an inspection plan for a problem with six periods. As shown in Figure 1, in the inspection plan, inspections are carried out at the beginning of the first, fourth, and sixth periods.

According to the definition, the last sentence in the sequence $I$ is always considered 1 ; therefore, it is shown differently from the rest. Consider the subsequence $\bar{I}=(1,1,1,1)$ that consists of ones in the sequence $I$ and sequence $k=(0,3,5,6)$ that contains the index of these ones.

The sequence of the stages corresponding to $I$ is obtained by:

$$
J(I)=(6-6,6-5,6-3,6-0)=(0,1,3,6) .
$$

This sequence shows that for the inspection plan $I=$ $(1,0,0,1,0,1,1)$, the stochastic dynamic programming model includes four stages: the first, second, third, and fourth stages show times when zero, one, three, and

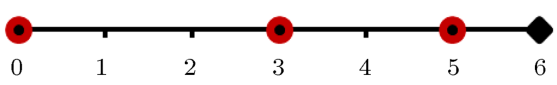

Figure 1. Inspection scheme for Example 1. 
six time periods remain until the end of the planning horizon, respectively.

\section{The second component (state variable)}

The machine status is defined as the state variable of the model and the indexes $s$ and $s^{\prime}$ are considered for it, such that $s, s^{\prime} \in \mathbb{S}$.

\section{The third component (decision variable)}

In our problem, there are two types of decision variables. The first one specifies the periods in which the machine should be inspected, while the second one specifies the period selected for the PM execution according to the state (machine status) of the current stage at each stage until the next inspection time (the next stage). Note that there is a possibility that the PM would not be executed in a period. Each inspection plan is a finite sequence of the first type of decision variables. Assume that $I=\left(i_{k}\right)_{k=0}^{K}$ is an arbitrary inspection plan and henceforth constant, and $J(I)=$ $\left(j(I)_{n}\right)_{n=0}^{m(I)}$ is the sequence of its corresponding stages in the dynamic stochastic programming model. In the stage $n$, the second-type decisions space corresponding to this inspection plan is $\mathbb{A}=\left\{0,1, \cdots, l_{I, n}\right\}$, such that $l_{I, n}=j(I)_{n}-j(I)_{n-1}$. In this space, decision 0 means that after the implementation of the inspection, the decision to perform preventive maintenance is not made, and decision $a$ means that the preventive maintenance will be implemented during the $a$ th period between the current and the next inspections $(1 \leq a \leq$ $\left.l_{I, n}\right)$.

IV. Fourth component (transition probability matrix) We assume that the machine deterioration process is a homogeneous continuous-time Markov process with a discrete state space $\mathbb{S}=\{0,1, \cdots, N\}$ shown by $\mathcal{X}=\left(X_{t}: t \in[0, \infty)\right)$. Having the transition rate matrix is enough to obtain the transition probability matrix of the process. The transition rate matrix $Q$ is defined as follows:

$$
\begin{aligned}
& Q=\left[q_{s s^{\prime}}\right]_{(N+1) \times(N+1),} \\
& q_{s s^{\prime}}=\lim _{t \rightarrow 0} \frac{\operatorname{Pr}\left(X_{t}=s^{\prime} \mid X_{0}=s\right)}{t}, \quad s, s^{\prime} \in \mathbb{S}, \quad s \neq s^{\prime}, \\
& q_{s s}=-\sum_{s^{\prime} \neq s} q_{s s^{\prime}} .
\end{aligned}
$$

Furthermore, we assume that the following conditions hold for the matrix $Q$ :

Condition 1: Without implementing PM, the machine status deteriorates because of production, i.e.:

$$
q_{s s^{\prime}}=0, \quad \forall s^{\prime}<s .
$$

Condition 2: The rate of transition to inferior states increments as a result of machine deterioration. In other words, we have:

$$
\sum_{s^{\prime} \geq u} q_{s s^{\prime}}<\sum_{s^{\prime} \geq u} q_{(s+1) s^{\prime}}, \quad \forall u \in \mathbb{S}, \quad u \geq(s+2) .
$$

Now, the transition probability matrix of the process is obtained using the Chapman-Kolmogorov equation [23]. Assume that $P_{0}$ and $P_{1}$ are these matrices after a time interval elapsing with length $T$ when PM is or is not carried out, respectively. Therefore, we have:

$$
\begin{aligned}
& P_{0}=e^{\mathcal{Q} T}, \\
& P_{1}=R \times P_{0}=R \times e^{\mathcal{Q} T},
\end{aligned}
$$

where $R$ is defined as follows:

$$
\begin{aligned}
& R=\left[r_{s s^{\prime}}\right]_{(N+1) \times(N+1)}, \\
& r_{s s^{\prime}}=\operatorname{Pr}\left(X_{0^{+}}=s^{\prime} \mid X_{0}=s, a=1\right) .
\end{aligned}
$$

As assumed above, the machine is restored to the asgood-as-new status following PM application:

$$
R=\left[\begin{array}{cccc}
1 & 0 & \cdots & 0 \\
\vdots & 0 & \cdots & 0 \\
1 & 0 & \cdots & 0
\end{array}\right] .
$$

V. Fifth component (total cost between two successive inspections). Assume that $C_{n, l}^{Z}(s, a)$ is the total cost between the current inspection and the next one (two successive inspections) in which $Z, n, s, l$, and a are demand vector, current inspection index in an inspection plan, machine status in the inspection, the number of time periods between the current inspection and the next one, and a member of decision space $\mathbb{A}=\{0,1, \cdots, l\}$, respectively. $C_{n, l}^{Z}(s, a)$ includes one inspection cost, one PM cost at most for all the periods between these two successive inspections, the expected value of the minimal repair cost, and the backlog cost in each period between these two successive inspections. It is calculated using Eqs. (6)-(8):

$$
\begin{aligned}
& C_{n, l}^{Z}(s, 0)=c_{i n s}+c_{m r} \int_{T_{1}(s, 0)}^{T} \lambda_{N-1} d t+h[z(n) \\
& \left.\quad-T_{A 1}(s, 0) r(s, 0)\right]^{+} \\
& \quad+\beta_{1}(l) \sum_{w=1}^{l-1} \rho^{w} \sum_{s^{\prime}=0}^{N}\left(P_{0}^{w}\right)_{s, s^{\prime}}\left\{c_{m r} \int_{T_{1}\left(s^{\prime}, 0\right)}^{T} q_{N-1} d t\right. \\
& \left.\quad+h\left[z(n+w)-T_{A 2}\left(s^{\prime}, 0\right) r\left(s^{\prime}, 0\right)\right]^{+}\right\} \\
& (n \in\{1, \cdots, K\}, \quad l \in\{1, \cdots, K-n+1\}, \quad s \in \mathbb{S}),
\end{aligned}
$$




$$
\begin{aligned}
& C_{n, l}^{Z}(s, 1)=c_{i n s}+c_{p m}(s)+c_{m r} \int_{T_{1}(s, 1)}^{T} \lambda_{N-1} d t \\
& +h\left[z(n)-T_{A 1}(s, 1) r(s, 1)\right]^{+} \\
& +\beta_{1}(l) \sum_{w=1}^{l-1} \rho^{w} \sum_{s^{\prime}=0}^{N}\left(P_{1} P_{0}^{w-1}\right)_{s, s^{\prime}} \\
& \left\{\begin{array}{l}
c_{m r} q_{N-1}^{T} d t+h[z(n+w) \\
T_{1}\left(s^{\prime}, 0\right) \\
\left.-T_{A 2}\left(s^{\prime}, 0\right) r\left(s^{\prime}, 0\right)\right]^{+}
\end{array}\right\}
\end{aligned}
$$$$
n \in\{1, \cdots, K\}, \quad l \in\{1, \cdots, K-n+1\}, \quad s \in \mathbb{S},
$$

$$
\begin{aligned}
& C_{n, l}^{Z}(s, a)=c_{i n s}+c_{m r} \int_{T_{1}(s, 0)}^{T} \lambda_{N-1} d t+h(z(n) \\
& \left.-T_{A 1}(s, 0) r(s, 0)\right)^{+}+\beta_{2}(a) \sum_{w=1}^{a-2} \rho^{w} \sum_{s^{\prime}=0}^{N}\left(P_{0}^{w}\right)_{s, s^{\prime}} \\
& \left\{c_{m r} \int_{T_{1}\left(s^{\prime}, 0\right)}^{T} q_{N-1} d t+h[z(n+w)\right. \\
& \left.\left.-T_{A 2}\left(s^{\prime}, 0\right) r\left(s^{\prime}, 0\right)\right]^{+}\right\}+\rho^{a-1} \sum_{s^{\prime}=0}^{N}\left(P_{0}^{a-1}\right)_{s, s^{\prime}} \\
& \left\{c_{p m}\left(s^{\prime}\right)+c_{m r} \int_{T_{1}\left(s^{\prime}, 1\right)}^{T} \lambda_{N-1} d t\right. \\
& \left.+h\left[z(n+a-1)-T_{A 2}\left(s^{\prime}, 1\right) r\left(s^{\prime}, 1\right)\right]^{+}\right\} \\
& +\beta_{a}(l) \sum_{w=a}^{l-1} \rho^{w} \sum_{k=0}^{N}\left(P_{0}^{a-1} P_{1} P_{0}^{w-a}\right)_{s, s^{\prime}} \\
& \left\{c_{m r} \int_{T_{1}\left(s^{\prime}, 0\right)}^{T} q_{N-1} d t+h[z(n+w)\right. \\
& \left.\left.-T_{A 2}\left(s^{\prime}, 0\right) r\left(s^{\prime}, 0\right)\right]^{+}\right\} \\
& a \in\{2, \cdots, K\}, \quad n \in\{1, \cdots, K\},
\end{aligned}
$$

$$
l \in\{a, \cdots, K-n+1\}, \quad s \in \mathbb{S} .
$$

Eq. (6) calculates the cost between two successive inspections in a situation where the current inspection index, machine status in the inspection, and the number of periods until the next inspection are $n$, $s, l$, respectively, and $\mathrm{PM}$ action is not implemented between these two inspections. Eq. (7) calculates this cost in similar conditions with the difference that PM action is implemented in the first period between those two inspections. If the number of periods between two successive inspections is greater than one, then Eq. (8) is used for calculating $C_{n, l}^{Z}(s, a)$ in the case of $\mathrm{PM}$ action within the $a$ th period of time.

In these equations, $\rho$ is a discount factor and $z(w)$ is the demand in the period $w . T_{A 1}(s, b)$ and $T_{A 2}(s, b)$ are actual times for production operations in one period in the case of performing and not performing the inspection, respectively, such that the machine is in the state $s$ at the beginning of the time period and action $b$ is decided for PM execution. These expressions are calculated as follows:

$$
T_{A 1}(s, b)=T-t_{\text {ins }}-b t_{p m}(s)-t_{m r} \int_{T_{1}(s, b)}^{T} \lambda_{N-1} d t,
$$$$
b \in\{0,1\}
$$

$$
T_{A 2}(s, b)=T-b t_{p m}(s)-t_{m r} \int_{T_{1}(s, b)}^{T} \lambda_{N-1} d t,
$$

$b \in\{0,1\}$.

At the above phrases, integral expression calculates the expected value of the number of failures within interval $\left[T_{1}(s, b), T\right]$.

From the first failure to the end of period, the process is changed into a homogenous Poisson process with state space $\mathbb{S}^{\prime}=\{N-1, N\}$, because we assumed the machine to be repaired minimally in case of unexpected failures. Therefore, the machine failure time has an exponential distribution with parameter $\lambda_{N-1}=-q_{N-1, N-1}[24]$. Hence, the expected value of the number of failures within interval $\left[T_{1}(s, b), T\right]$ is calculated as follows:

$$
\int_{T_{1}(s, b)}^{T} \lambda_{N-1} d t
$$

where:

$$
T_{1}(s, b)=\min \left\{\kappa_{N}((1-b) s), T\right\} .
$$

In addition, $\kappa_{N}((1-b) s)$ is the expected value of the hitting time to failure state $(N)$ from state $(1-b) s$ (i.e., the state 0 if $\mathrm{PM}$ is executed and the state $s$ 
otherwise) calculated by solving the following equation system [25]:

$$
\begin{cases}\kappa_{N}(s)=0, & s \in\{N\} \\ \sum_{s^{\prime}} q_{s s^{\prime}} \kappa_{N}\left(s^{\prime}\right)=-1, & s \in \mathbb{S} \backslash\{N\}\end{cases}
$$

The $r(s, b)$ is the machine production rate in one period such that the machine state is $s$ at the beginning of the time period and action $b$ is decided for PM execution:

$$
r(s, b)= \begin{cases}r(s) & b=0 \\ r(0) & b=1\end{cases}
$$

$\beta_{h}$ is an indicator function such that:

$$
\begin{aligned}
& \beta_{h}:\{h, \cdots, K\} \rightarrow\{0,1\} \\
& \beta_{h}(l)= \begin{cases}0 & l=h \\
1 & l \geq h+1\end{cases}
\end{aligned}
$$

\subsubsection{Optimality equation of the proposed model}

In this section, by considering the definition of the optimality equation components provided in the previous section, an optimality equation is constructed for each inspection plan. For this purpose, suppose that $I=\left(i_{k}\right)_{k=0}^{K}$ is an arbitrary inspection plan and henceforth constant, and $J(I)=\left(j(I)_{n}\right)_{n=0}^{m(I)}$ is its corresponding stage sequence. Then, Eq. (9) shows the optimality equation of the proposed model:

$$
\begin{aligned}
V_{j(I)_{n}}(s)= & \min _{a \in\left\{0,1, \cdots, l_{I, n}\right\}}\left\{C_{\bar{j}_{n}, l_{I, n}}^{Z}(s, a)\right. \\
& \left.+\rho^{l_{I, n}} \sum_{s^{\prime}=0}^{N}(\mathcal{P}(a))_{s, s^{\prime}} V_{j(I)_{n-1}}\left(s^{\prime}\right)\right\} \\
n \in\{1, \cdots, m(I)\} &
\end{aligned}
$$

where:

$$
\begin{aligned}
& \bar{j}_{n}=K-j(I)_{n}+1, \quad l_{I, n}=j(I)_{n}-j(I)_{n-1}, \\
& V_{j(I)_{0}}(s)=0 \quad s \in \mathbb{S}, \\
& P(a)= \begin{cases}P_{0}^{l_{I, n}} & a=0 \\
P_{0}^{a-1} P_{1} P_{0}^{l_{I, n}-a} & a \in\left\{1,2, L, l_{I, n}\right\}\end{cases}
\end{aligned}
$$

\section{Solution method}

To solve the presented models, the following four-step algorithm is employed.

Step 1: For each element of $\mathbb{I}$ as $I=\left(i_{k}\right)_{k=0}^{K}$ (i.e., an arbitrary inspection plan and henceforth constant) execute Steps 2 and 3;
Step 2: Make the stage sequence $J(I)=\left(j(I)_{n}\right)_{n=0}^{m(I)}$ corresponding to the inspection plan $I=\left(i_{k}\right)_{k=0}^{K}$;

Step 3: For each state $s$, the optimality equation, i.e., a backward recursive equation, is used to calculate the optimal value and find the corresponding optimal decision and, then, name them $V_{j(I)_{m(I)}}^{*}(s)$ and $a_{I}^{*}(s)$, respectively. In other words, $a_{I}^{*}(s)$ is the optimal decision on the time of executing PM actions at the interval between the first and second inspections when the inspection plan is $I$ and the system state at the beginning of time horizon is $s$;

Step 4: For each state $s$, select the inspection plan with the minimum value $V_{j(I)_{m(I)}}^{*}(s)$ as the optimal inspection plan corresponding to $s$ in the planning horizon and name it $I^{*}=\left(i_{k}^{*}\right)_{k=0}^{K}$;

Step 5: For each state of $s$ and for the optimal inspection plan $I^{*}$ corresponding to $s$, select $a_{I^{*}}^{*}(s)$ as an optimal decision for PM execution between the first and second inspections.

\section{Numerical study}

In this section, an illustrative example is designed to analyze the strategy of the proposed model and to investigate the effect of taking demand into account in the model.

Designing an illustrative example to analyze the strategy of the proposed model is explained in this section while the effect of demand is investigated simultaneously.

\subsection{Detailed example}

Suppose that the planning horizon consists of 6 periods, each of which is 30 days long, the machine states space is $\mathbb{S}=\{0,1,2,3,4\}$, and the discount factor is one. Other parameters are listed in Tables 13. Relation (10) shows the transition rate matrix that applies to Relations (2) and (3) to satisfy the Conditions 1 and 2. The one-step transition matrices

Table 1. Backlog, inspection, and minimal repair parameters.

\begin{tabular}{ccccc}
\hline $\boldsymbol{h}(\$)$ & $\boldsymbol{c}_{\text {ins }}(\$)$ & $\boldsymbol{t}_{\text {ins }}($ day) & $\boldsymbol{c}_{\boldsymbol{m} \boldsymbol{r}}(\$)$ & $\boldsymbol{t}_{\boldsymbol{m} \boldsymbol{r}}$ (day) \\
\hline 5 & 400 & 1 & 640 & 1 \\
\hline
\end{tabular}

Table 2. Test problems demand.

\begin{tabular}{lcccccc}
\hline \multirow{2}{*}{ Demand } & \multicolumn{6}{c}{ Period, time no. } \\
\cline { 2 - 7 } & $\mathbf{1}$ & $\mathbf{2}$ & $\mathbf{3}$ & $\mathbf{4}$ & $\mathbf{5}$ & $\mathbf{6}$ \\
\hline Low & 100 & 150 & 200 & 180 & 300 & 250 \\
Medium & 300 & 360 & 432 & 475 & 523 & 575 \\
High & 1200 & 1300 & 1100 & 1500 & 1000 & 1200 \\
Nominal & 600 & 600 & 600 & 600 & 600 & 600 \\
\hline
\end{tabular}


Table 3. PM and production rate parameters depending on machine state.

\begin{tabular}{cccc}
\hline $\boldsymbol{s}$ & $\boldsymbol{c}_{\boldsymbol{p m}}(\boldsymbol{s})$ & $\boldsymbol{t}_{\boldsymbol{p m}}(\boldsymbol{s})$ & $\boldsymbol{r}(\boldsymbol{s})$ \\
\hline 0 & 0 & 0 & 20 \\
1 & 300 & 1 & 16 \\
2 & 500 & 2 & 10 \\
3 & 900 & 3 & 2 \\
4 & 1500 & 4 & 0 \\
\hline
\end{tabular}

are calculated in the absence and presence of $\mathrm{PM}$ execution via Eqs. (4) and (5), respectively, and shown in Eqs. (11) and (12):

$$
\begin{aligned}
& \mathcal{Q}=\left[\begin{array}{ccccc}
-0.100 & 0.040 & 0.020 & 0.030 & 0.010 \\
0.000 & -0.107 & 0.041 & 0.031 & 0.035 \\
0.000 & 0.000 & -0.107 & 0.032 & 0.075 \\
0.000 & 0.000 & 0.000 & -0.094 & 0.094 \\
0.000 & 0.000 & 0.000 & 0.000 & 0.000
\end{array}\right] \\
& P_{0}=\left[\begin{array}{lllll}
0.050 & 0.054 & 0.059 & 0.101 & 0.736 \\
0.000 & 0.041 & 0.050 & 0.072 & 0.837 \\
0.000 & 0.000 & 0.040 & 0.047 & 0.913 \\
0.000 & 0.000 & 0.000 & 0.059 & 0.941 \\
0.000 & 0.000 & 0.000 & 0.000 & 1.000
\end{array}\right] \\
& P_{1}=\left[\begin{array}{lllll}
0.050 & 0.054 & 0.059 & 0.101 & 0.736 \\
0.050 & 0.054 & 0.059 & 0.101 & 0.736 \\
0.050 & 0.054 & 0.059 & 0.101 & 0.736 \\
0.050 & 0.054 & 0.059 & 0.101 & 0.736 \\
0.050 & 0.054 & 0.059 & 0.101 & 0.736
\end{array}\right]
\end{aligned}
$$

\subsection{Results analysis}

In this section, the effectiveness of demand counting in the performance of the proposed model is considered. For this purpose, the solution algorithm was implemented by MATLAB software first. Then, the numerical example was designed under two situations. In the first situation, the demand was eliminated from the model by replacing zero instead of the unit backlog cost $(h)$. Consequently, in this situation, we deal with a pure CBM model. Instead of the second one, the unit backlog cost was substituted by $5(h=5)$ and demand was considered. This model was run by four different demand vectors, namely low, medium, high, and nominal demands, separately. The model under this situation is named demand-driven CBM. Table 4 shows the optimal inspection plans for the first and second situations.

These results indicate that the inspection plan $I_{1}$, which is the optimal solution of the pure CBM model for all states, is not optimal for all states in the demand-driven CBM model and instead, $I_{16}$ or $I_{32}$ is the optimal inspection plan depending on the machine status at the beginning of the planning horizon and the demand vector. In addition, in the demand-driven CBM model, higher demand or worse machine status increases the number of inspections.

Tables 5 shows the optimal solution to execute the PM based on the state of the system at the inspection time corresponding to the first and second situations. For example, in the pure CBM model, first, the inspection plan of $I_{1}$ is optimal; second, in the first inspection, if the system state is 2,3 , or 4, the first period is the best for the PM execution; otherwise, PM should not be executed. In the demanddriven CBM model, the optimal interval of the PM depends on the demand vector. For instance, in the medium demand case and in the first inspection, if the system state is 2 , first, the inspection plan of $I_{16}$ is optimal. Second, the optimal decision for preventive maintenance is $a=2$, meaning that the PM must be carried out in the second period of the ones between the first and second inspection periods (period 2).

Then, in other inspections, depending on the machine state, PM must be carried out in the same inspection period $(a=1)$ or must not be executed $(a=0)$. Moreover, these solutions indicate that if the machine state is worsened or the demand rises, the number of inspections increases and the preventive maintenance should be executed at the same time or earlier.

Table 6 shows the optimal inspection plan and the optimal total cost for the demand-driven CBM model and the optimal CBM cost for the pure CBM model depending on the machine state at the beginning of the planning horizon (initial machine state). The inspection plans were found optimal in at least one of the models while other inspection plans were discarded. Besides, the optimal costs were shown in bold. These results show that:

1. Toward the aforementioned parameters, the inspection plan $I_{1}$ is optimal for the pure CBM model independent of the initial machine state;

2. The optimal inspection plan $I_{1}$ is not necessarily optimal for the demand-driven CBM model;

3. The optimum inspection plan obtained by the demand-driven CBM model leads to the reduction of the total cost in comparison to the optimum inspection plan obtained by the pure CBM model. The amount of this reduction depends on the demand vector and the initial machine state. This reduction is called the total improvement value displayed in percentage in Table 2. For example, if the initial state is 2 and the demand vector is medium, then the improvement value is $30.3 \%$;

4. As a managerial insight, if the demand vector components are the nearby nominal capacity $(600=$ 
Table 4. Optimal inspection plans.

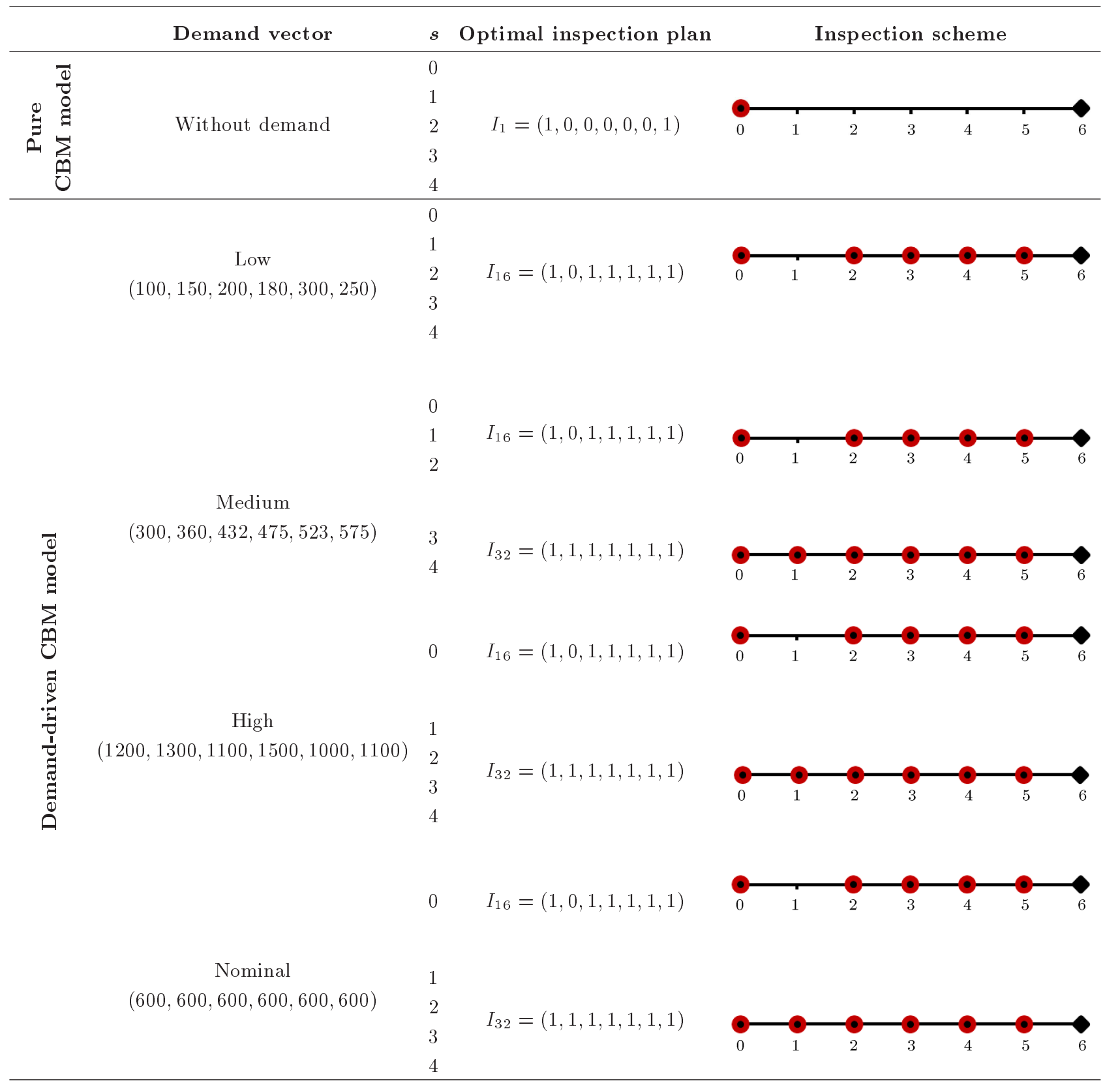

$30 \times 20$ ), then the demand-driven CBM model is more efficient.

Consequently, enumerating demand in a CBM model leads to a significant reduction in the total costs. Furthermore, this model can provide operational managers with a roadmap to overcome the trade-off between CBM costs and backlog cost, which is an inescapable part of production costs.

\subsection{Sensitivity analysis and discussion}

The sensitivity of the total improvement value to the unit backlog cost is analyzed in this section after running more than 100 different unit backlog costs.
The objective is to find out which demand-driven CBM model conditions would yield better revenue. Accordingly, for each initial machine state and demand vector, the pure and demand-driven CBM models were solved for different values of the unit backlog cost $(h)$. Then, the total improvement value corresponding to each of them was calculated. Figures 2 to 6 show that:

1. The total improvement value is an increasing and concave function in unit backlog cost $(h)$ regardless of the initial machine state and the demand vector. The positivity trait of the function shows that considering demand in the modeling leads to 
Table 5. Optimal PM execution interval corresponding to each of the situations.

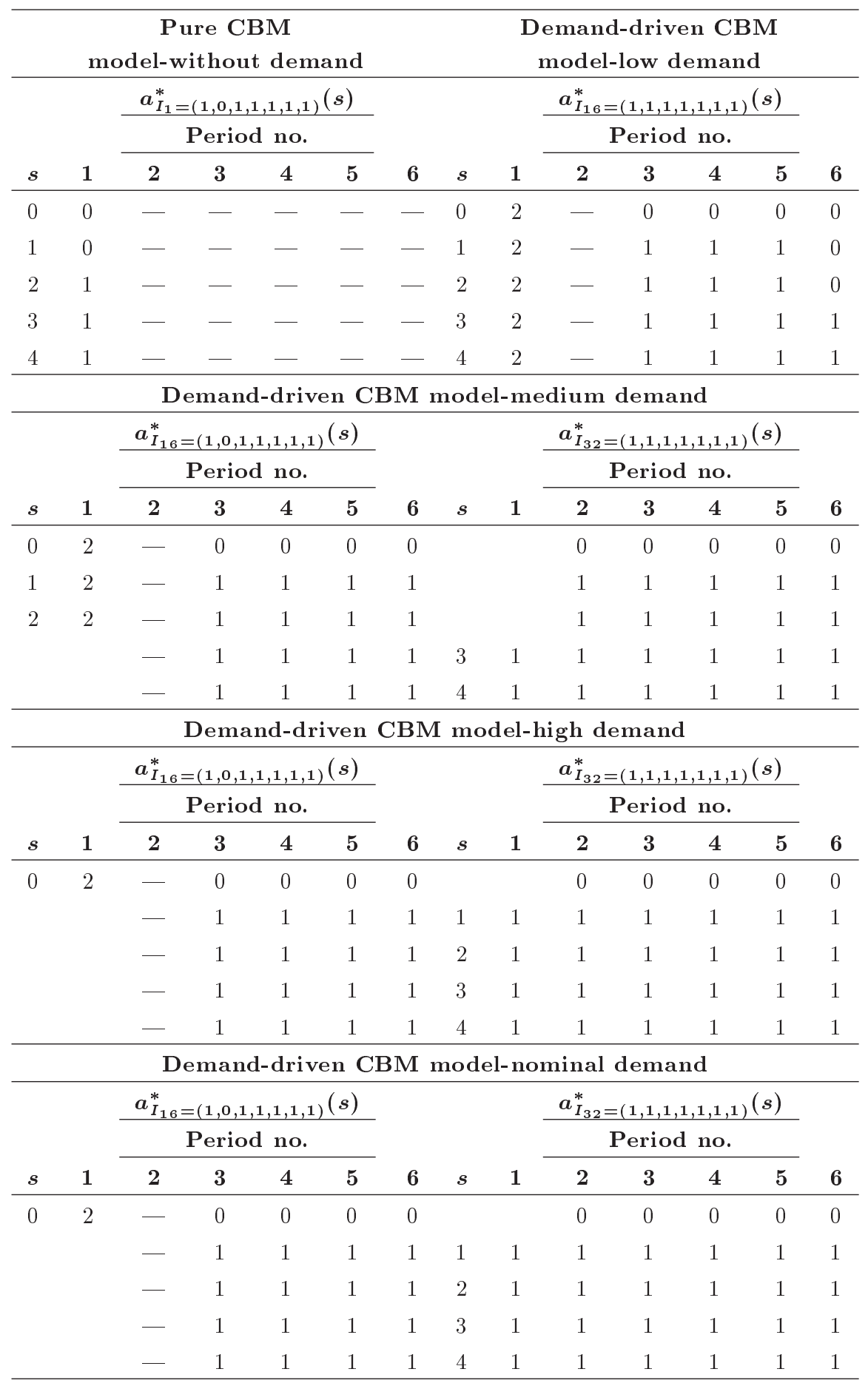

adopting a more optimized CBM policy rather than ignoring the demand. Meanwhile, it is shown that a greater increase in the unit backlog cost leads to greater improvement by employing the proposed CBM policy;

2. Comparison of an arbitrary demand vector with different initial states illustrates that improvement sensibility is negligible. Moreover, comparing an ar- bitrary initial state with different demands implies adopting a CBM policy with a sensible improvement. Therefore, the operational managers need to consider demand rather than the machine initial state. In other words, demand has more impact on the improvement of total cost than the initial machine state.

3. When the unit backlog cost $(h)$ is greater than a 
Table 6. Optimal inspection plan and cost for pure CBM and demand-driven CBM models.

\begin{tabular}{|c|c|c|c|c|c|c|}
\hline \multirow{2}{*}{$\begin{array}{c}\text { Initial } \\
\text { machine } \\
\text { state (s) }\end{array}$} & \multirow{2}{*}{$\begin{array}{c}\text { Optimal } \\
\text { inspection } \\
\text { plan }\end{array}$} & \multirow{2}{*}{$\begin{array}{c}\text { Pure } \\
\text { CBM } \\
\text { cost }\end{array}$} & \multicolumn{4}{|c|}{ Demand-driven CBM cost } \\
\hline & & & $\begin{array}{c}\text { Low } \\
\text { demand }\end{array}$ & $\begin{array}{l}\text { Medium } \\
\text { demand }\end{array}$ & $\begin{array}{c}\text { High } \\
\text { demand }\end{array}$ & $\begin{array}{l}\text { Nominal } \\
\text { demand }\end{array}$ \\
\hline \multirow{3}{*}{0} & $I_{1}=(1,0,0,0,0,0,1)$ & 9608 & 13309 & 18429 & 40168 & 21668 \\
\hline & $I_{16}=(1,0,1,1,1,1,1)$ & 11208 & 12209 & 12820 & 33561 & 15061 \\
\hline & \multicolumn{2}{|c|}{ Total improvement value } & $8.3 \%$ & $30.4 \%$ & $16.4 \%$ & $30.5 \%$ \\
\hline \multirow{4}{*}{1} & $I_{1}=(1,0,0,0,0,0,1)$ & 9908 & 13740 & 18942 & 41332 & 22982 \\
\hline & $I_{16}=(1,0,1,1,1,1,1)$ & 11508 & 12576 & 13186 & 34546 & 16046 \\
\hline & $I_{32}=(1,1,1,1,1,1,1)$ & 11905 & 12909 & 13520 & 34461 & 15961 \\
\hline & \multicolumn{2}{|c|}{ Total improvement value } & $8.5 \%$ & $30.4 \%$ & $16.6 \%$ & $30.6 \%$ \\
\hline \multirow{4}{*}{2} & $I_{1}=(1,0,0,0,0,0,1)$ & 10094 & 13921 & 19303 & 42416 & 23916 \\
\hline & $I_{16}=(1,0,1,1,1,1,1)$ & 11694 & 12716 & 13453 & 35535 & 17035 \\
\hline & $I_{32}=(1,1,1,1,1,1,1)$ & 12094 & 13109 & 13720 & 34761 & 16261 \\
\hline & \multicolumn{2}{|c|}{ Total improvement value } & $8.7 \%$ & $30.3 \%$ & $18.0 \%$ & $32.0 \%$ \\
\hline \multirow{4}{*}{3} & $I_{1}=(1,0,0,0,0,0,1)$ & 10249 & 14332 & 20615 & 43725 & 25225 \\
\hline & $I_{16}=(1,0,1,1,1,1,1)$ & 11849 & 13106 & 14716 & 36804 & 18304 \\
\hline & $I_{32}=(1,1,1,1,1,1,1)$ & 12249 & 13506 & 14120 & 35261 & 16761 \\
\hline & \multicolumn{2}{|c|}{ Total improvement value } & $8.6 \%$ & $31.5 \%$ & $19.4 \%$ & $33.6 \%$ \\
\hline \multirow{4}{*}{4} & $I_{1}=(1,0,0,0,0,0,1)$ & 10929 & 15303 & 21585 & 44695 & 26195 \\
\hline & $I_{16}=(1,0,1,1,1,1,1)$ & 12529 & 14054 & 15664 & 37552 & 19052 \\
\hline & $I_{32}=(1,1,1,1,1,1,1)$ & 12929 & 14109 & 14720 & 35961 & 17461 \\
\hline & \multicolumn{2}{|c|}{ Total improvement value } & $8.2 \%$ & $31.8 \%$ & $19.5 \%$ & $33.3 \%$ \\
\hline
\end{tabular}

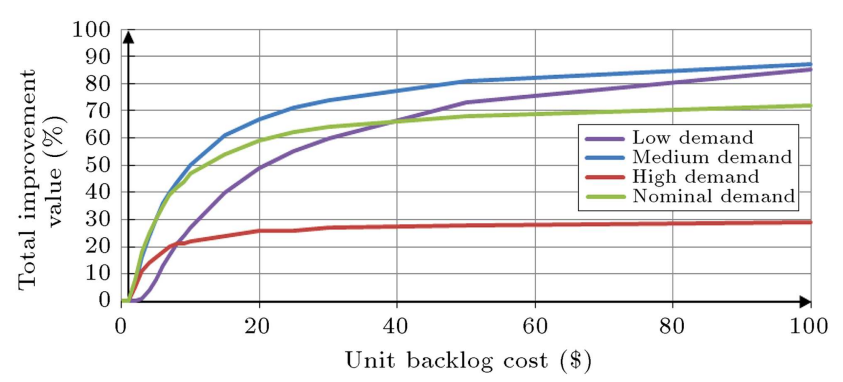

Figure 2. The total improvement value as a function of the unit backlog in state 0 .

certain amount, the total improvement value does not grow considerably. It is implied that by increasing the unit backlog cost, the improvement of the total cost increases slowly and, therefore, knowing the real unit backlog cost plays an important role

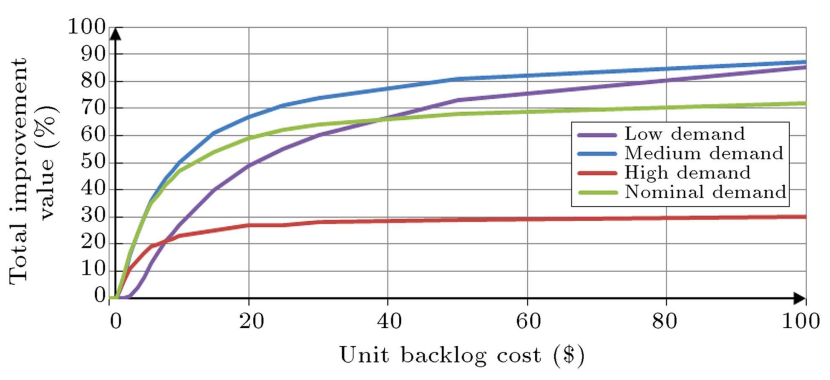

Figure 3. The total improvement value as a function of the unit backlog in state 1 .

in the structure of this model. Therefore, by considering the low demand, the total improvement value is highly dependent on the unit backlog cost. However, in case of high or medium demand, this dependence will not be too considerable. In general, 


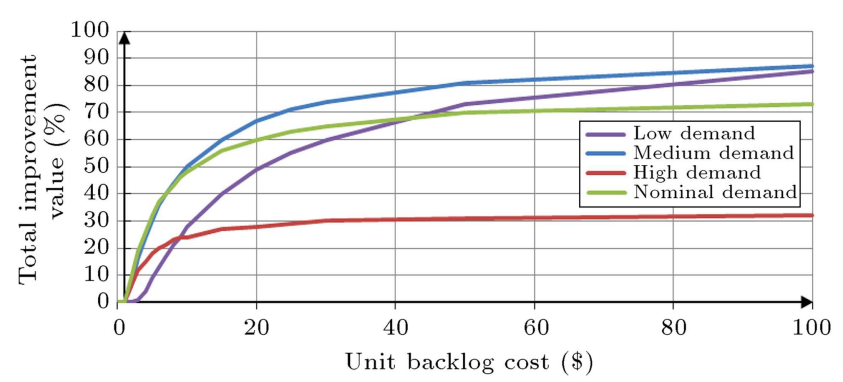

Figure 4. The total improvement value as a function of the unit backlog in state 2 .

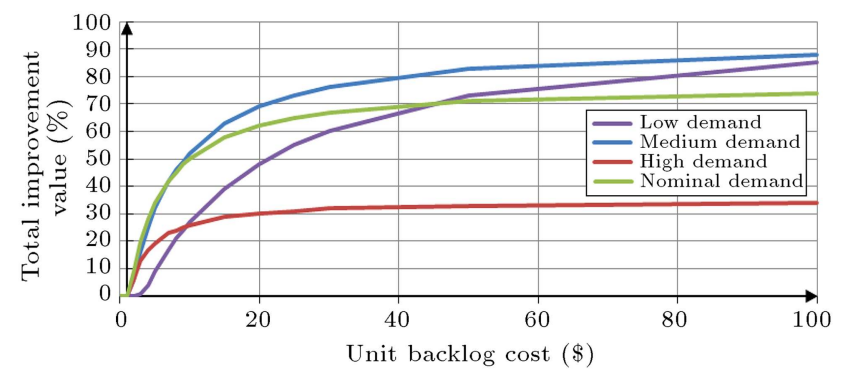

Figure 5. The total improvement value as a function of the unit backlog in state 3 .

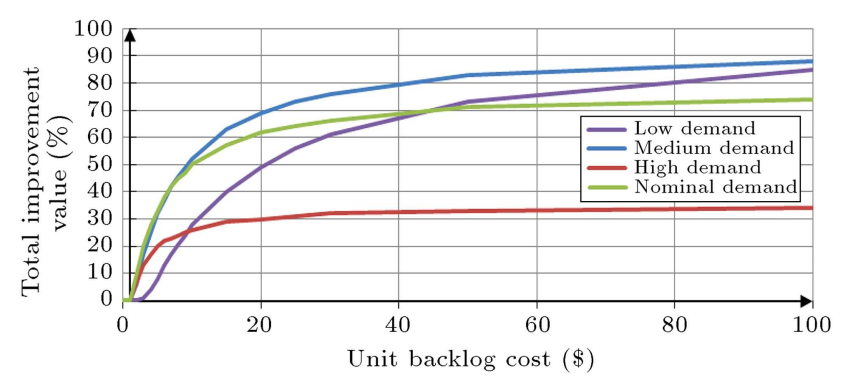

Figure 6. The total improvement value as a function of the unit backlog in state 4 .

estimating the real demand and unit backlog cost is very important in adopting a suitable CBM policy.

\section{Conclusion}

In this research, CBM decisions consisting of the determination of suitable periods for inspection and $\mathrm{PM}$ execution in single-product single-machine systems were dependent on customer demand, while the machine deterioration process was modeled by applying a continuous-time Markov chain. These decisions were adopted to minimize the sum of inspection, PM, repair, and backlog costs in which it is assumed that the machine was minimally repaired upon failure and PM was conducted to restore it to the 'as-good-as-new' status. The stochastic dynamic process framework was employed to achieve this purpose.

The main contribution of this research is that inspection and PM decisions were dependent on customer demand. This dependency initially prevented unneces- sary inspection and PM operations, which were costly and caused customer dissatisfaction due to delays in orders. Secondly, it provided a basis for the integration of production and condition-based maintenance planning. Moreover, managers, generally limited in maintenance budgets, would save considerable costs.

To solve the problem, all of the inspection schemes were generated, for each of which the optimality equation of the model was solved via a back-ward recursive algorithm. Finally, an optimal combination of the inspection period and PM period was selected.

To analyze the effect of demand on the total cost, the proposed model was solved in two situations. In the first situation, by replacing zero instead of the unit backlog cost, a pure CBM model was run. Instead, in the second one, i.e., demand-driven CBM model, the unit backlog cost was considered and demand was taken into account. This model was run by four different demand vectors, namely low, medium, high, and nominal demands, separately. The numerical results illustrated that regardless of the initial machine state, taking the demand into account by the CBM model would reduce the total costs. Of course, this reduction depends on the demand vector and the lost production unit cost. This demand-driven CBM model could be employed for each data collection and any lifetime distribution. In the end, for future research, it is recommended that other decisions of aggregation production planning be incorporated into the proposed models of this research. Moreover, establishing sufficient conditions that guarantee the monotonicity in both machine status and demand for the problem with a similar situation in an infinite time horizon can be interesting.

\section{References}

1. Aramon Bajestani, M. "Integrating maintenance planning and production scheduling: Making operational decisions with a strategic perspective", A Thesis for Ph.D. degree, University of Toronto (2014).

2. Aramon Bajestani, M., Banjevic, D., and Beck, J.C. "Integrated maintenance planning and production scheduling with Markovian deteriorating machine conditions", International Journal of Production Research, 52(24), pp. 7377-7400 (2014).

3. Wang, H. "A survey of maintenance policies of deteriorating systems", European Journal of Operational Research, 139(3), pp. 469-489 (2002).

4. Ahmad, R. and Kamaruddin, S. "An overview of timebased and condition-based maintenance in industrial application", Computers \& Industrial Engineering, 63(1), pp. 135-149 (2012).

5. Alaswad, S. and Xiang, Y. "A review on conditionbased maintenance optimization models for stochasti- 
cally deteriorating system", Reliability Engineering \& System Safety, 157, pp. 54-63 (2017).

6. Jardine, A.K., Lin, D., and Banjevic, D. "A review on machinery diagnostics and prognostics implementing condition-based maintenance", Mechanical Systems and Signal Processing, 20(7), pp. 1483-1510 (2006).

7. Golmakani, H.R. "Condition-based inspection scheme for condition-based maintenance", International Journal of Production Research, 50(14), pp. 3920-3935 (2012).

8. Golmakani, H.R. and Fattahipour, F. "Age-based inspection scheme for condition-based maintenance", Journal of Quality in Maintenance Engineering, 17(1), pp. 93-110 (2011).

9. Lam, J.Y.J. and Banjevic, D. "A myopic policy for optimal inspection scheduling for condition-based maintenance", Reliability Engineering \& System Safety, 144, pp. 1-11 (2015).

10. Mishra, A. and Jain, M. "Maintainability policy for deteriorating system with inspection and common cause failure", International Journal of Engineering, Transaction C: Basics, 26(6), pp. 371-380 (2013).

11. Xu, M., Alam, M.N.E., and Kamarthi, S. "A modified dynamic programming model in condition-based maintenance optimization", 12th International Manufacturing Science and Engineering Conference, Los Angeles, California, USA, pp. V003T04A014-V003T04A014 (2017).

12. Banjevic, D., Jardine, A.K.S., Makis, V., et al. "A control-limit policy and software for condition-based maintenance optimization", INFOR: Information Systems and Operational Research, 39(1), pp. 32-50 (2001).

13. Liu, B., Wu, S., Xie, M., and Kuo, W. "A conditionbased maintenance policy for degrading systems with age-and state-dependent operating cost", European Journal of Operational Research, 263(3), pp. 879-887 (2017).

14. Yazdanparast, S., Sadegheih, A., Fallahnezhad, M., and Abooie, M. "Modelling and decision-making on deteriorating production systems using stochastic dynamic programming approach", International Journal of Engineering, 31(12), pp. 2052-2058 (2018).

15. Ghandali, R., Abooie, M.H., and Nezhad, F. "A POMDP framework to find optimal inspection and maintenance policies via availability and profit maximization for manufacturing systems", International Journal of Engineering, 31(12), pp. 2077-2084 (2018).

16. Sloan, T.W. "A periodic review production and maintenance model with random demand, deteriorating equipment, and binomial yield", Journal of the $O p$ erational Research Society, 55(6), pp. 647-656 (2004).

17. Sloan, T.W. and Shanthikumar, J.G. "Combined production and maintenance scheduling for a multipleproduct, single-machine production system", Production and Operations Management, 9(4), pp. 379-399 (2000).

18. Sloan, T.W. "Simultaneous determination of production and maintenance schedules using in-line equipment condition and yield information", Naval Research Logistics (NRL), 55(2), pp. 116-129 (2008).

19. Kang, K. and Subramaniam, V. "Integrated control policy of production and preventive maintenance for a deteriorating manufacturing system", Computers \& Industrial Engineering, 118, pp. 266-277 (2018).

20. Kang, K. and Subramaniam, V. "Joint control of dynamic maintenance and production in a failureprone manufacturing system subjected to deterioration", Computers \& Industrial Engineering, 119, pp. 309-320 (2018).

21. Aghezzaf, E.H., Khatab, A., and Le Tam, P. "Optimizing production and imperfect preventive maintenance planning's integration in failure-prone manufacturing systems", Reliability Engineering \& System Safety, 145, pp. 190-198 (2016).

22. Ross, S.M., Introduction to Stochastic Dynamic Programming, pp. 49-50, Academic press, New York, USA (1983).

23. Ross, S.M., Introduction to Probability Models, 11th Edn., pp. 396-397, Academic Press, Amsterdam, Netherlands (2010).

24. Ross, S.M., Introduction to Probability Models, 11th Edn., pp. 383-384, Academic Press, Amsterdam, Netherlands (2010).

25. Berestycki, N. and Sousi P., Applied Probability, pp. 21-22 (2015).

\section{Biographies}

Reza Noori is a PhD Student of Industrial Engineering at Yazd University in Iran. He received his BSc and MSc degrees in Pure Mathematics and Applied Mathematics in Operations Research from Kharazmi University and Amirkabir University in 2005 and 2008, respectively. His research interest areas include mathematical modelling, maintenance planning, and optimization under uncertainty. He has published a number of papers in journals and conferences.

Ahmad Sadegheih is a Professor of Industrial Engineering at the College of Engineering, Yazd University in Iran. He obtained his $\mathrm{PhD}$ degrees in Industrial Engineering from University of Wales Cardiff in UK (1999). He serves as the Head of Industrial Engineering Faculty at Yazd University. His research interests 
include operations research, linear programming, integer programming, dynamic programming, system engineering, decision analysis, genetic algorithms, engineering economy, management and economic, Multiple Criteria Decision-Making (MCDM), Analytical Hierarchy Process (AHP), Goal Programming (GP), and meta heuristic algorithms. He has been a project leader for some projects and has published more than 200 journal and conference papers.

Mohammad Mehdi Lotfi is an Associate Professor of Industrial Engineering at Yazd University. His areas of expertise are manufacturing and service operations management. His research interests include optimization, decision tools and quantitative methods in operations management \& logistics (especially, humanitarian logistics), and healthcare. He has been a project manager for many projects and has published more than 50 journal papers. He received his BS degree from Sharif University of Technology and his MS and PhD degrees from University of Tehran. 